\title{
A rare disease with a rare presentation: hemi-atrophy caused by fibromuscular dysplasia in a 27 month old girl
}

\author{
B. Gharib ${ }^{1,2}$, A. Ghareh Zadeh Shirazi' ${ }^{1}$ E. Moradi ${ }^{3}$, B. Yaghmaei ${ }^{1,5}$, V. Ziaee ${ }^{1,4,5}$ \\ ${ }^{1}$ Children's Medical Center, Pediatrics Center of Excellence, Tehran; 'Growth and Development research \\ Center, Tehran University of Medical Sciences, Tehran; ${ }^{3}$ Shiraz University of Medical Sciences, Shiraz; \\ ${ }^{4}$ Pediatric Rheumatology Research Group, Rheumatology Research Center, Tehran University of Medical \\ Sciences, Tehran; ${ }^{5}$ Department of Pediatrics, Tehran University of Medical Sciences, Tehran, Iran
}

\begin{abstract}
SUMMARY
We discuss the case of a 27-month-old girl afflicted with fibromuscular dysplasia. She presented with hemiatrophy of left upper and lower limbs, nail dystrophy, ulcers on the tips of her toes, cold and painful limbs, foot drop, and hypertension. The initial appearance started at 2 months of age and other diagnoses such as complex regional pain syndrome, reflex sympathetic syndrome, vasculitis and coagulation disorders had been considered. Angiography revealed that all the arterial branches of the left lower and upper limbs, from brachial to ulnar and radial, and from iliac and femoral to tibialis arteries were affected. Sural nerve biopsy confirmed the diagnosis. In the follow-up visits until 2 years after the patient's discharge she did not develop any new problem and her blood pressure was controlled by enalapril and amlodipine.
\end{abstract}

Key words: Fibromuscular dysplasia; Hemiatrophy; Childhood; Hypertension.

Reumatismo, 2019; 71 (1): 31-36

\section{INTRODUCTION}

ibromuscular dysplasia (FMD) is a non-inflammatory arterial involvement that appears with hypertension and neuralgic symptoms. Medium size arteries such as renal and carotid arteries are the main vascular involvement, but every artery can get involved (1). Involvement of the other arteries such as intracranial, basilar, axillary, iliac and hepatic have also been reported. The etiology of FMD is not known (2).

FMD usually affects women of 20-60 years of age; however it may occur in men and, more rarely, in infants, children and elderly. In this paper we report a patient with FMD who suffered from numbness and hemiatrophy from 2 months of age.

\section{CASE REPORT}

A 27-month-old girl was referred to our hospital to evaluate her hemiatrophy. Her left upper and lower limbs were smaller than the limbs of the other side. Her weight was 11 kilograms (less than $3^{\text {rd }}$ percentile), her height was 92 centimeters (between 75 and 90 percentile).

The problem started when she was 2 months of age and her mother attributed the problem to routine vaccinations (Diphtheria, Tetanus, Pertussis, Hepatitis B, and oral Poliomyelitis) that she received at this age. Her mother noticed mottling, occasional cyanosis, and relative coldness in the left lower limb, a few days after receiving the vaccine. The vaccines were injected to her left thigh. The above-mentioned symptoms were aggravated by cold weather and water exposure (even moderate temperature) and accompanied with restlessness. The signs and symptoms were constant and not paroxysmal. She had not received any other vaccine since then, in consideration of the possible immunodeficiency.

On the affected side (left side), her nails were dystrophic, and compared to the other side the length and circumferences of the limbs were smaller (Fig. 1-3), the limbs
Corresponding author: Vahid Ziaee Division of Pediatric Rheumatology Children's Medical Center, No. 62 Dr. Gharib St. Keshavarz Blvd, Tehran 14194, IR Iran E-mail: ziaee@tums.ac.ir 


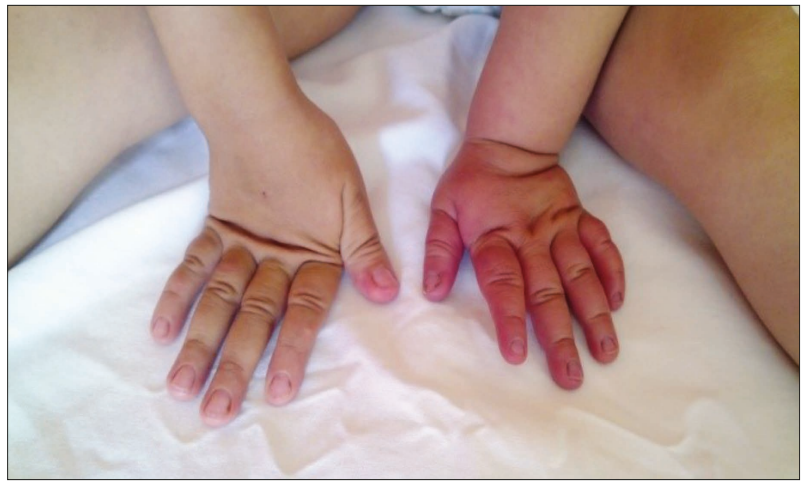

Figure 1 - The size and length of the left hand is smaller than the other side and the nails appear dystrophic.

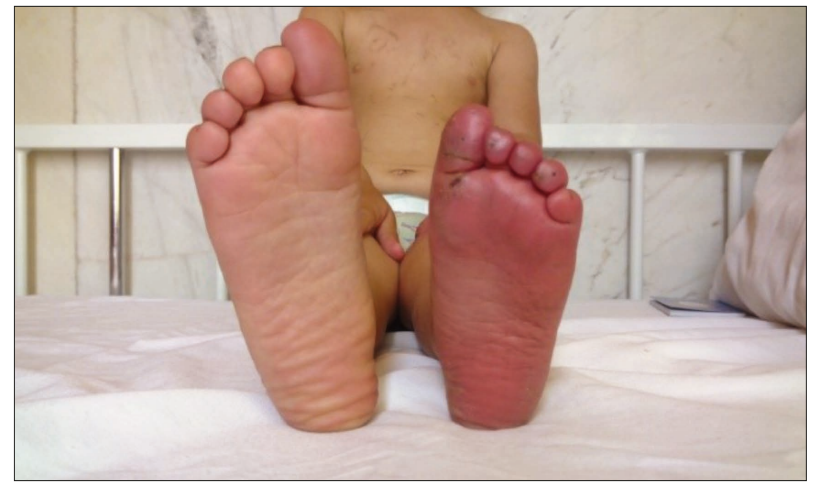

Figure 2 - Left foot is smaller than the right foot, with ulcers at the tip of the toes. were colder and the peripheral pulses were significantly weaker. There had been occasional ulcers at the tip of the toes and fingers (Fig. 2). Her mother told us that after 2.5 years, she had not cut the patient's nails for a long time, as they had not grown normally. At the age of 2.5 years, she was limping on left side and the skin of the affected side was thinner. She had left foot drop since 3 weeks, after a few days of hospitalization.

Auscultation of heart, abdomen (abdominal aorta and renal arteries), neck (carotid and vertebral arteries), and lungs were normal. Abdominal examination was unremarkable. She suffered from hypertension, which had been detected at 18 months of age. Her blood pressure was 170/100 $\mathrm{mmHg}$ (measured from the right arm) on admission. Blood pressure was not measured from the affected limbs as they were very sensitive to pain (allodynia).

In the past medical history, her birth weight was 4 kilograms, she was born by cesarean section at 38 weeks of gestation because of vaginal bleeding and meconium aspiration. Her parents were non-consanguineous. She was admitted in different medical centers and several differential diagnoses such as immunodeficiency, coagulation disorders (she had received heparin), antiphospholipid syndrome, vasculitis (she received high dose methylprednisolone for 2 days), and viral hepatitis were considered, she did not have immunodeficiency and all related workups were concluded with normal results.
Ten months prior to the current admission a computerized tomography angiography reported as follows; Normal left axillary artery, significant narrowing in the mid and distal part of the brachial artery, abrupt

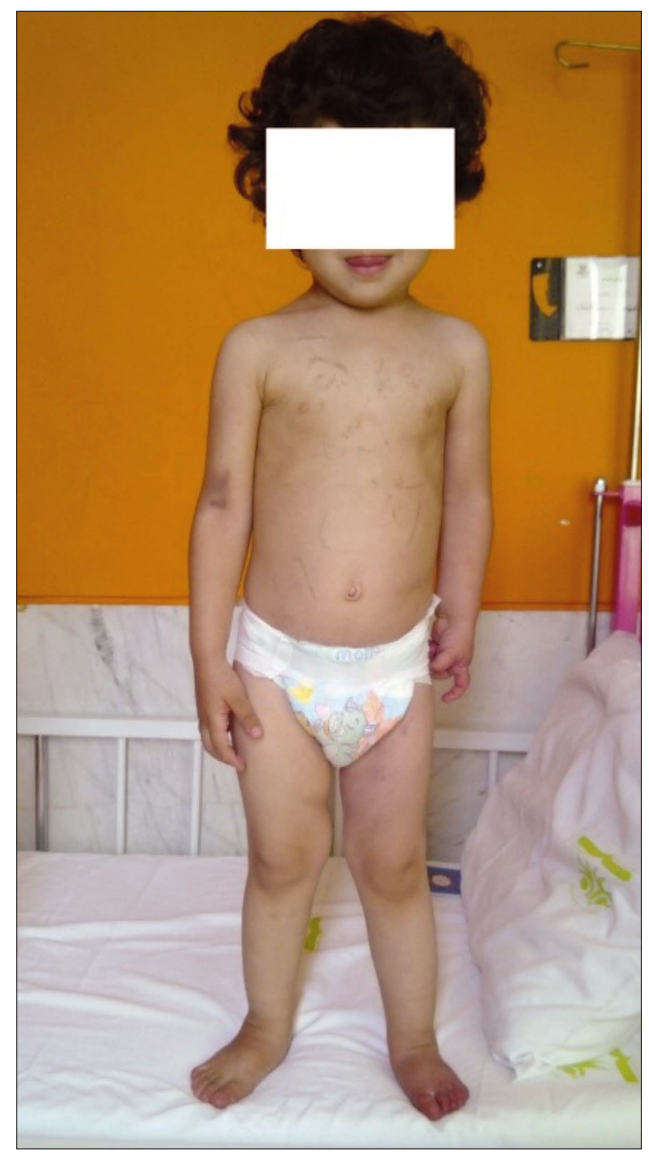

Figure 3 - The left side limbs are shorter and smaller than the other side. 
cut off at the level of anterior cubital area in the brachial artery before bifurcation. Distal to the mentioned site, radial and ulnar arteries were not visible. Left common iliac artery, left external iliac and left common femoral artery and superficial femoral artery were smaller than the right side. Narrowing of the left popliteal artery was seen. Narrowing of the left posterior tibialis was seen and distal part of the left popliteal artery was not opacified. Left anterior tibialis artery was not seen (Fig. 4).

At 18 months of age she was evaluated in a tertiary university hospital. Diagnoses of reflex sympathetic dystrophy or complex regional pain syndrome were suggested and the neurosurgeon consultant recommended performing sympathetic block. Left thoracoscopic cervicothoracic sympathectomy and open left lumbar sympathectomy were performed. The temperature of the left limbs increased afterward but this was a temporary favorable effect that lasted for just a few weeks.

The laboratory work-up results were normal except for plasma renin $=37.7 \mu \mathrm{IU} / \mathrm{mL}$ (reference rage $=0.06-4.69 \mu \mathrm{IU} / \mathrm{mL}$ ) and aldosterone $=746 \mathrm{pg} / \mathrm{mL}$ (reference range; early morning supine $=20-180 \mathrm{pg} / \mathrm{mL}$, upright $2 \mathrm{~h}=30-400 \mathrm{pg} / \mathrm{mL}$ ).

At 27 months of age, the bone age (measured by plain wrist radiography) of the right hand was reported as 24 months, and in the left hand (the affected side) as 15 months.

Ultrasonography of kidneys showed that the length of the right kidney was shorter $(68 \mathrm{~mm})$ than the left kidney $(84 \mathrm{~mm})$. Echogenicity of both kidneys was normal. Electromyography and nerve conduction velocity of the left extremities suggested considering small fiber neuropathy. Transthoracic echocardiography showed left ventricular hypertrophy and tricuspid valve regurgitation. Abdominal ultrasonography was normal.

She was administered enalapril tablets 5 $\mathrm{mg} /$ day/twice a day, aspirin $5 \mathrm{mg} / \mathrm{kg} /$ day $/$ twice a day, hydralazine infusion $25 \mathrm{~mL}$ in the case that the blood pressure measurement was equal to or more than $130 / 90$ $\mathrm{mmHg}$, cilostazol tab $25 \mathrm{mg}$ twice a day (for upper and lower limbs ischemia).

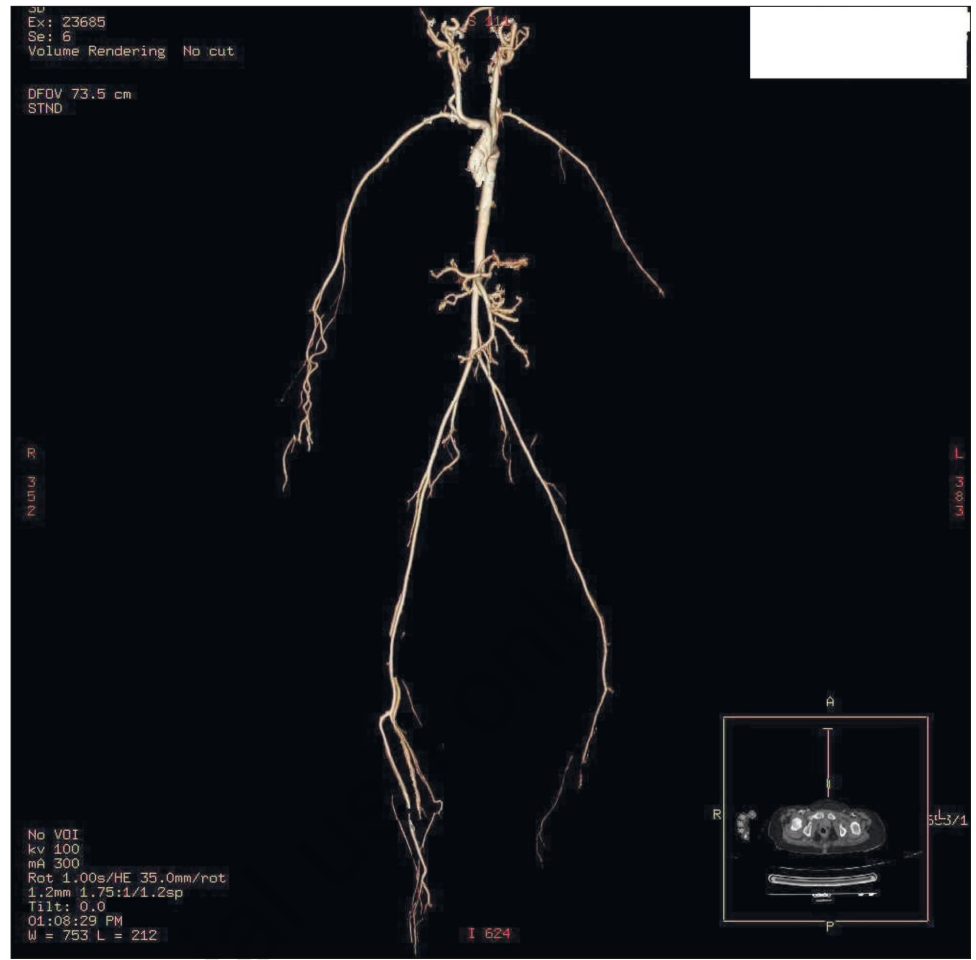

Figure 4 - The computerized tumor angiography revealed significant narrowing in the following arteries of left upper extremity: mid and distal part of the brachial artery, anterior cubital area in the brachial artery before bifurcation, radial and ulnar arteries, and also in the following arteries of the left lower extremity: common iliac, external iliac, common femoral, superficial femoral, popliteal, posterior tibialis, distal part of the popliteal artery, and anterior tibialis artery.

A biopsy of the sural nerve was performed with the following result:

- Fibrous thickening, irregular medial epineurium (fibromuscular dysplasia), and small artery.

- Peripheral neuropathy mainly axonal with secondary myelin break down and minimal focal nerve fiber loss (angiopathic neuropathy).

She was discharged with enalapril tablets, $2.5 \mathrm{mg}$ twice a day, and amlodipine, $2.5 \mathrm{mg}$ twice a day, hydralazine tablets, $2.5 \mathrm{mg}$, in the case that her blood pressure measured $130 / 90 \mathrm{mmHg}$ or more. In addition, she was under treatment with aspirin tablets (50 mg daily) as anti-platelet aggregation therapy and nifedipine tablets, $5 \mathrm{mg}$ for Raynaud's phenomena twice a day. During a one-year follow up, hydralazine was discontinued and hypertension was controlled 
with increase dosage of amlodipine to 2.5 $\mathrm{mg}$ three times daily and enalapril tablets $2.5 \mathrm{mg}$ twice a day. In the follow-up visits until 3 years after the patient's discharge she has not developed any new problem and her blood pressure has been controlled by enalapril and nifedipine.

\section{DISCUSSION AND CONCLUSIONS}

Fibromuscular dysplasia (FMD) was described by Leadbetter and Burkland $(1,3)$ in a 5.5 year old boy with severe hypertension (3) in 1938 for the first time $(1,3)$. Our patient presented it in early infancy, which is very rare for FMD and, based on our review of the literature, this case is the youngest patient with FMD that has been reported in the literature (4-15).

The pediatric population with FMD show more frequent family history of FMD than adults (13). In $6-10 \%$ of familial FMDs, autosomal dominant transmission beside incomplete penetrance along with variable expressivity is proposed and no specific gene has been found yet (12). FMD has not been associated with gene polymorphism. Associations between genetic mutations attributed with connective tissue disorders, such as the COL31A gene, transforming growth factor $(T G F)-\beta 1$ and $\beta 2$ genes, and the ACTA2 gene had been imperceptible (14). It seems there is no a major gene for FMD and multifocal FMD may have been associated with MYLK, OBSCN, DYNC2H1 and RNF213 genes (15).

Transient ischemic attack (TIA), dissection or stroke may occur when FMD involves carotid or vertebral arteries. FMD may cause stenosis, dissection, obstruction and aneurysm but many patients with FMD are asymptomatic. In persons with cervical bruit, a whooshing sound in the ear, stroke, TIA, dissection of an artery, onset of hypertension at or below 35 years of age, FMD should be considered (1). If FMD affects renal arteries, hypertension is the most common presentation. Our patient had renal artery involvement in the right kidney. According to the United States Regis- try for FMD, all children with FMD have hypertensione, and abdominal bruits and headache are common presentations, renal vessels are the most commonly involved and mesenteric and abdominal aorta are also often affected. Cervical bruits are not frequent $(5.3 \%)$ and no coronary involvement is reported. About $7 \%$ of the affected children had bruits over the epigastrium, carotid and flanks [epigastrium (7.4\%), carotid arteries $(7.4 \%)$, and flanks $(7.7 \%)$ ] but not on the femoral arteries. However, in adults $34.6 \%$ had carotid bruits. The most prevalent sign in children was renovascular hypertension. In adults, pulsatile tinnitus, neck pain and carotid artery dissection have been reported significantly more commonly (13).

FMD in cervical and cranial vessels may cause dissection and then present as headache, strokes, Horner's syndrome, intracranial aneurysm and hemorrhage (2). FMD mainly affects medium-sized arteries (4). In the general population, systemic hypertension is not common in childhood, and usually is secondary to background disease and problems. Encephalopathy caused by hypertension with signs and symptoms of dizziness, nausea, poor activity and unsteady gait have been reported in FMD (5). FMD may show the same presentation of necrotizing vasculitis or remain without any symptoms. It can cause mesenteric ischemia or claudication. In children younger than 4 years of age, FMD is likely to be similar to vasculitis. Ehlers-Danlos syndrome type 4, Marfan, Alport, and pheochromocytoma are among the conditions associated with FMD (6). FMD can lead to strokes in children with systemic arteriopathy and hypertension (7). Intimal fibroplasia of the right coronary artery associated with fatty infiltration of the right ventricular myocardium has been reported as the cause of sudden death in children (8). Arteries dissection is generally the reason for acute limb ischemia in iliac FMD. Iliac FMD may cause bruit, heard from the umbilicus to the inguinal region. Brachial arteries are the most commonly involved arteries in the upper extremities (3). In the majority of FMD cases (more than $80 \%$ ), 
multifocal stenosis with the string-ofbeads are seen in angiography (2).

Differentiating FMD from vasculitis can be difficult and sometimes patients were treated for primary vasculitis. Our patient was evaluated for vasculitis twice but we didn't find any trace of vasculitis in para clinical findings. However, she was treated as for primary vasculitis and/or antiphospholipid syndrome by pulse therapy with methylprednisolone but we didn't find significant response to treatment. Large-vessel vasculitis can manifest without alterations in acute-phase reactants in up to $40 \%$ of patients and their angiographic appearance may be similar (9). Vascular Ehlers-Danlos and Williams' syndromes, type 1 neurofibromatosis, atherosclerotic or inflammatory artery diseases are the differential diagnosis of arterial stenosis caused by FMD (2). Because the first presentation of our case, the patient was considered to have other diseases, such as reflex sympathetic dystrophy and complex regional pain syndrome. Side effects of BCG vaccine were also suggested.

The diagnosis of FMD is made mostly on the basis of the angiographic. Based on the layer involved of the arteria wall (intima, media, adventitia), FMD is pathologically classified (10). Adventitial FMD is the least prevalent type of FMD. In the extremities, external iliac arteries are the most commonly involved. Involvement of arteries below the inguinal ligament are uncommon. FMD of external iliac artery is often asymptomatic, however claudication or, more rarely, acute limb ischemia may occur (3). In our case, CT-angiography showed that all the arterial branches of the lower and upper limbs, from brachial to ulnar and radial and from iliac and femoral to tibialis arteries, had been affected and had led to atrophic limbs, dystrophic nails, and finger and toe ulcers. In our patient, the diagnosis was made by sural nerve biopsy.

Treatment options for FMD include antiplatelet therapy, balloon angioplasty, and stent placement or surgical repair of aneurysm (1). European consensus on the diagnosis and management of fibromuscular dysplasia in 2014, has recommended revascularization as a treatment for hypertensive adult patients with FMD-related renal artery stenosis (RAS) who have hypertension of recent onset (as a first line of treatment), medical therapy failure, renal insufficiency or degradation of renal function specifically after prescription of angiotensin converting enzyme inhibitor, angiotensin 2 receptor blocker or a renin inhibitor, and in cases of reduction of renal size caused by FMD (11). In this patient, signs and symptoms were managed by nifedipine, enalapril, aspirin and cilostazol and occasional hydralazine.

We suggest that FMD should be considered in hypertensive children especially when no other reason has been identified.

Conflict of interest: the authors declare they have no conflicts of interest.

\section{REFERENCES}

1. Olin JW, Sealove BA. Diagnosis, management, and future developments of fibromuscular dysplasia. J Vasc Surg. 2011; 53: 826-36.

2. Plouin PF, Perdu J, La Batide-Alanore A, et al. Fibromuscular dysplasia. Orphanet J Rare Dis. 2007; 2: 28.

3. Olin JW, Gornik HL, Bacharach JM, et al. Fibromuscular dysplasia: state of the science and critical unanswered questions: a scientific statement from the American Heart Association. Circulation. 2014; 129: 1048-78.

4. Çomoglu S, Gürer Y. Early onset stroke due to fibromuscular dysplasia in a child. Turk J Med Sci. 2006; 36: 251-3.

5. Chen M, Lee H, Chang W, et al. Hypertensive encephalopathy caused by fibromuscular dysplasia of renal arteries in a child, stenosis. Case Rep Acta Cardiol. Sin. 2012; 28: 60-2.

6. Olin JW. Recognizing and managing fibromuscular dysplasia. Cleve Clin J Med. 2007; 74: 273-4, 277-82.

7. Kirton A, Crone M, Benseler S, et al. Fibromuscular dysplasia and childhood stroke. Brain. 2013; 136: 1846-56.

8. Osterheld M, Perez M. Two unusual cases of sudden death in children. Open J Pathol. 2013; 3: 69-72.

9. Slovut DP, Olin JW. Fibromuscular dysplasia. N Engl J Med. 2004; 350; 1862-71.

10. Touzé E, Oppenheim C, Trystram D, et al. Fibromuscular dysplasia of cervical and intracranial arteries. Int J Stroke. 2010; 5: 296-305. 
11. Persu A, Giavarini A, Touzé E, et al. European consensus on the diagnosis and management of fibromuscular dysplasia. J Hypertens. 2014; 32: 1367-78.

12. Varennes L, Tahon F, Kastler A, et al. Fibromuscular dysplasia: what the radiologist should know: a pictorial review. Insights Imaging. 2015; 6: 295-307.

13. Green R, Gu X, Kline-Rogers E, et al. Differences between the pediatric and adult presentation of fibromuscular dysplasia: results from the US Registry. Pediatr Nephrol. 2016; 31: 641-50.

14. Olin JW, Gornik HL, Bacharach JM, et al; on behalf of the American Heart Association Council on Peripheral Vascular Disease, Council on Clinical Cardiology, Council on Cardiopulmonary, Critical Care, Perioperative and Resuscitation, Council on Cardiovascular Disease in the Young, Council on Cardiovascular Radiology and Intervention, Council on Epidemiology and Prevention, Council on Functional Genomics and Translational Biology, Council for High Blood Pressure Research, Council on the Kidney in Cardiovascular Disease, and Stroke Council. Fibromuscular dysplasia: state of the science and critical unanswered questions: a scientific statement from the American Heart Association. Circulation. 2014; 129: 1048-78.

15. Kiando SR, Barlassina C, Cusi D, et al. Exome sequencing in seven families and genebased association studies indicate genetic heterogeneity and suggest possible candidates for fibromuscular dysplasia. J Hypertens. 2015; 33: 1802-10. 